Check for updates

Cite this: Phys. Chem. Chem. Phys., 2017, 19, 13449

Received 3rd March 2017 Accepted 26th April 2017

DOI: $10.1039 / c 7 c p 01404$

rsc.li/pccp

\section{Molecular organization in the twist-bend nematic phase by resonant $X$-ray scattering at the Se K-edge and by SAXS, WAXS and GIXRD $\dagger$}

\author{
W. D. Stevenson, ${ }^{\text {a }}$ Z. Ahmed, ${ }^{\mathrm{b}}$ X. B. Zeng, (D)*a C. Welch, ${ }^{\mathrm{b}}$ G. Ungar ${ }^{* a c}$ and \\ G. H. Mehl*b
}

Using a magnetically aligned liquid crystal mixture containing a novel Se-labelled dimer and the difluoroterphenyl dimer DTC5C7, the twist-bend nematic phase $\left(\mathrm{N}_{\mathrm{tb}}\right)$ was studied by the resonant scattering of hard X-rays and by conventional small and wide-angle X-ray scattering (SAXS, WAXS). Resonant diffraction spots indicated a helix with a 9-12 $\mathrm{nm}$ pitch in the $\mathrm{N}_{\mathrm{tb}}$ phase and an unprecedentedly high helix orientation. This enabled deconvolution of global and local order parameters. These findings, combined with the simultaneously recorded resonant and non-resonant SAXS and WAXS data, allowed us to construct a locally layered molecular model of the $\mathrm{N}_{\mathrm{tb}}$ phase, where the average twisted conformation of each molecule was idealised as a helical segment, matching the local heliconical director field. The dimers were found to be less bent in the $N_{t b}$ phase than in their minimum energy conformation, and straightening further with increasing temperature. It is proposed that on further heating their low bend angle allows the transition to the normal nematic phase, where the molecules can freely move longitudinally, without the need to perform screw-like motion as in the $\mathrm{N}_{\mathrm{tb}}$ phase. At the low-temperature end, the increasing molecular twist becomes unsustainable, leading to a transition to a smectic phase, where no twist is required.

The nematic-nematic liquid crystal (LC) transition, first reported in main-chain LC polyethers ${ }^{1,2}$ and later in bent LC dimers, ${ }^{3-22}$ is commonly observed when mesogens are angled to each other (bent conformation) by a semi-flexible spacer, such as an oddnumbered oligomethylene chain. Prediction of the nematicnematic transition in $1976^{23}$ predates even the first experimental observation in $1992,{ }^{1}$ but the more recent theoretical framework provided by Dozov ${ }^{24}$ and Memmer ${ }^{25}$ has become the basis of reference. On transition from the higher temperature nematic $(\mathrm{N})$, it is proposed that the mesogens preferentially select a lower energy ground state with a locally bent director field, thereby

\footnotetext{
${ }^{a}$ Department of Materials Science and Engineering, University of Sheffield, Sheffield S1 3JD, UK. E-mail: x.zeng@shef.ac.uk

${ }^{b}$ Department of Chemistry, University of Hull, Hull HU6 $7 R X, U K$

'Department of Physics, Zhejiang Sci-Tech University, Hangzhou 310018, China

$\dagger$ Electronic supplementary information (ESI) available. See DOI: 10.1039/c7cp01404j
}

enabling a closer packing of bent molecular conformations. To fill space, the local director bend is accompanied by twist or splay deformations, giving rise to two alternative bent nematic ground states: helical twist-bend nematic $\left(\mathrm{N}_{\mathrm{tb}}\right)$ and oscillating splay-bend nematic $\left(\mathrm{N}_{\mathrm{sb}}\right) \cdot{ }^{24}$ Of importance to this work is the $\mathrm{N}_{\mathrm{tb}}$ model. Currently, the $\mathrm{N}_{\mathrm{tb}}$ phase is described as a nematic continuum featuring an average heliconical director field. The mesogens are thought to glide fluidly along the helical axis, possess no long-range positional order and complete a full rotation in just a few molecular lengths. Both left- and righthanded domains are expected.

Historically, the $\mathrm{N}_{\mathrm{tb}}$ model has proven difficult to unambiguously validate by experimental means. Topological characterisation methods, e.g. opto-electric studies, ${ }^{3,4}$ have shown inversely handed domains and switching speeds consistent with a nano-scale pitch. A nano-scale pitch was also suggested by $\mathrm{AFM}^{5}$ and freeze fracture TEM; ${ }^{6,7}$ though in both cases, the imaging was performed below the stability range of the $\mathrm{N}_{\mathrm{tb}}$ phase, and doubts remained as to whether the surface imaging was representative of the bulk. The structure of the $\mathrm{N}_{\mathrm{tb}}$ phase was also unclear from NMR, ${ }^{8}$ Raman scattering ${ }^{9}$ and conventional X-ray scattering. ${ }^{10}$ However, recently, helical periodicity within the $\mathrm{N}_{\mathrm{tb}}$ phase was directly identified in a bulk sample by resonant X-ray scattering at the carbon absorption edge (K-edge). ${ }^{11}$ A resonant Bragg ring was observed in the $\mathrm{N}_{\mathrm{tb}}$ phase originating from an 8-10 nm long helical pitch. Such observation of orientational periodicity is possible only at the absorption edge of the atoms, where X-ray scattering becomes sensitive to the symmetry of bound orbitals, i.e. where the atomic form factor changes from scalar to a complex tensor. ${ }^{26-28}$

Although the helical pitch has now been confirmed by carbon $\mathrm{RXS}$, many questions about the $\mathrm{N}_{\mathrm{tb}}$ phase remain unanswered. The three principal subjects of debate regard the orientational order parameter, the manner in which the molecules pack into the helix and whether or not the molecules are locally layered. However, carbon RXS is unable to provide answers to these questions, because the low energy 'soft' X-rays $(\sim 0.3 \mathrm{keV})$ required for carbon resonance imposes severe experimental constraints; for example, the beamline must be fully windowless in high 
vacuum and the sample must be held between thin, fragile substrates, preventing alignment by external fields. ${ }^{11}$ Moreover, soft X-ray scattering allows access to only a limited range of reciprocal space, excluding the scattering at larger wave vectors $(q)$, which holds information on molecular conformation and packing.

Here, we avoided the drawbacks of carbon RXS by studying the $\mathrm{N}_{\mathrm{tb}}$ phase using harder X-rays at the selenium K-edge (12.658 keV). For this purpose, a selenoether dimer was specially synthesised. In addition to resonant diffraction from the heliconical $\mathrm{N}_{\mathrm{tb}}$ structure, we extend the previous work by simultaneously recording the nonresonant scattering at larger $q$. Furthermore, we aligned the $\mathrm{N}_{\mathrm{tb}}$ phase using a magnetic field and, for the first time, obtained direct information on the orientation of the helical axis and, simultaneously, that of the molecules. This enabled us to obtain new details about the molecular conformation and packing in the $\mathrm{N}_{\mathrm{tb}}$ phase. Combined resonant and non-resonant small- and wideangle X-ray scattering (SAXS and WAXS) data on the perfectly aligned sample, together with the results from grazing incidence X-ray diffraction (GIXRD), allowed us to construct a more detailed model of the $\mathrm{N}_{\mathrm{tb}}$ phase. This includes twisted molecular conformations matching the shape of the local heliconical director field, as well as local molecular layering. We also present herein the first quantitative data on the helical domain size in bulk $\mathrm{N}_{\mathrm{tb}}$.

\section{Sample preparation and phase behaviour}

The selenoether dimer compound DTSe (Fig. 1a) was synthesised as described in the ESI. $\dagger$ DTSe does not exhibit the $\mathrm{N}_{\mathrm{tb}}$ phase, but did so when mixed with the previously studied compound DTC5C7 (Fig. 1a). ${ }^{12,13}$ The miscibility of the two compounds was examined qualitatively by bringing them into contact under crossed polarisers. A composition gradient was formed, which was most evident at $114{ }^{\circ} \mathrm{C}$. Fig. $1 \mathrm{~b}$ shows the central $\mathrm{N}$ phase separating the $\mathrm{N}_{\mathrm{tb}}$ phase of the DTC5C7-rich region (left) and the Smectic-C (SmC) phase of the DTSe-rich region (right).

The binary phase diagram (Fig. 1c) was determined by differential scanning calorimetry (DSC) and polarised optical microscopy. Each mixture was prepared by dissolving weighed amounts of DTC5C7 and DTSe in dichloromethane and freeze drying after mixing in solution. The diagram shows a stable $\mathrm{N}_{\mathrm{tb}}$ region between 0 and ca 60 mol\% DTSe. For our RXS investigation we selected a mixture containing 45 mol\% DTSe, henceforth referred to as Se45.

\section{Resonant X-ray scattering}

The RXS investigation of Se45 was performed on beamline I22 of the Diamond Light Source. The sample was held in a $1 \mathrm{~mm}$ glass capillary, positioned perpendicular to a $1 \mathrm{~T}$ magnetic field. The sample was heated and cooled using thermostated nitrogen gas. Se45 was investigated in each of its phases using $\mathrm{X}$-ray energies above and below the Se K-edge $\left(E_{\mathrm{r}}=12.658 \mathrm{keV}\right)$. Two Pilatus $2 \mathrm{M}$ detectors were simultaneously employed to

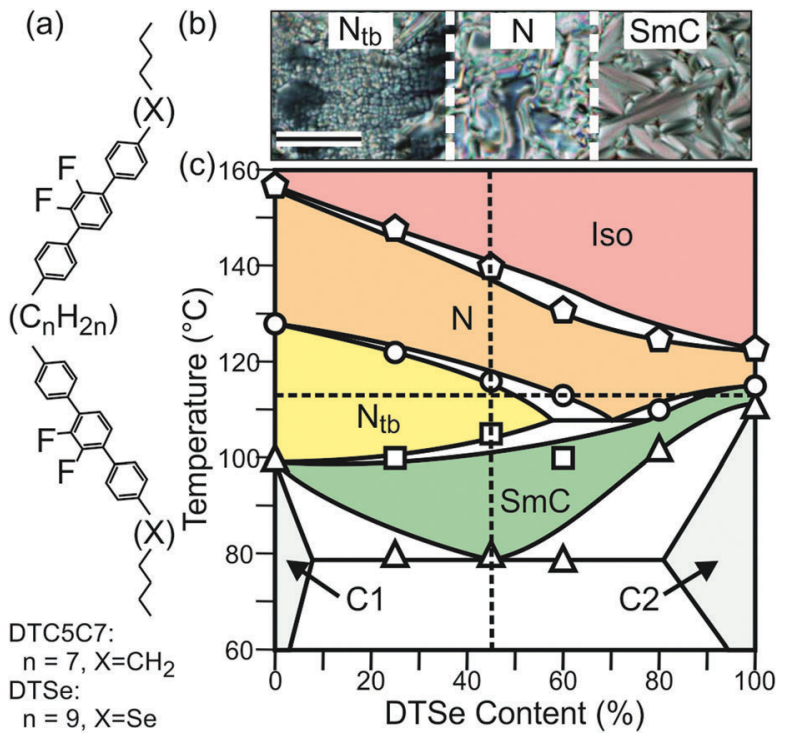

Fig. 1 (a) The molecular structure of DTC5C7 $\left(n=7, \mathrm{X}=\mathrm{CH}_{2}\right)$ and DTSe ( $n=9, X=S e$ ). (b) Optical textures observed between cross polarizers at $114{ }^{\circ} \mathrm{C}$ for a contact preparation, showing the change from $\mathrm{N}_{\mathrm{tb}}$, to $\mathrm{N}$, to $\mathrm{SmC}$ phase with increasing concentration of DTSe in the binary mixture. Scale bar $=100 \mu \mathrm{m}$. (c) Phase diagram of DTC5C7 and DTSe. The experimental points are the DSC peak temperatures on first heating (note: a metastable smectic forms in pure DTC5C7 on cooling ${ }^{12}$ ). C1 = DTC5C7 crystal, C2 = DTSe crystal. The dashed vertical line indicates Se45, and the horizontal line $114^{\circ} \mathrm{C}$.

cover the range of $\sim 0.3 \mathrm{~nm}^{-1} \leq q \leq 15 \mathrm{~nm}^{-1}$. Additional nonresonant SAXS and WAXS experiments were carried out at station BM28 (ESRF) using a MAR165 CCD camera.

The sample was first cooled from the isotropic to the $\mathrm{N}_{\mathrm{tb}}$ phase $\left(105{ }^{\circ} \mathrm{C}\right)$, where the incident energy $\left(E_{\mathrm{i}}\right)$ was increased in increments of $5 \mathrm{eV}$ from 12.608 to $12.708 \mathrm{keV}$. Throughout this energy range, two broad SAXS maxima were observed at $q=1.44$ and $3.16 \mathrm{~nm}^{-1}$, corresponding to average spacings of $4.4(d 1)$ and $2.0 \mathrm{~nm}(d 2)$ (Fig. S5 and Table S2, ESI $\dagger$ ). These spacings are comparable to the average (DTSe and DTC5C7) dimer length $(4.5 \mathrm{~nm})$ and centre-to-centre mesogen distance $(2.1 \mathrm{~nm})$ within Se45. These two SAXS maxima are believed to arise from localised smectic-like layering, usually driven by $\pi$ - $\pi$-interactions between aromatic mesogens and often referred to as cybotactic nematic correlations. ${ }^{29}$ A non-resonant diffuse WAXS maximum at $q=13.7 \mathrm{~nm}^{-1}$ corresponds to a " $d$-spacing" of $0.46 \mathrm{~nm}$, suggesting an average lateral intermolecular distance of $0.51 \mathrm{~nm} .{ }^{29}$ However at X-ray energies satisfying $\left|E_{\mathrm{i}}-E_{\mathrm{r}}\right| \leq 5 \mathrm{eV}$, an additional Bragg peak was observed at $q=0.62 \mathrm{~nm}^{-1}$, attributed to resonant diffraction from a helical pitch $p$ of $10.1 \mathrm{~nm}$ (Fig. S5, ESI $\dagger$ ). The resonant peak was strongest at $E_{\mathrm{i}}=E_{\mathrm{r}}$.

\section{Temperature dependence of the resonance effect}

Temperature dependence of the resonance effect was investigated by cooling the sample through each of its LC phases with $E_{\mathrm{i}}=E_{\mathrm{r}}$. The resonant peak was observed exclusively in the $\mathrm{N}_{\mathrm{tb}}$ 

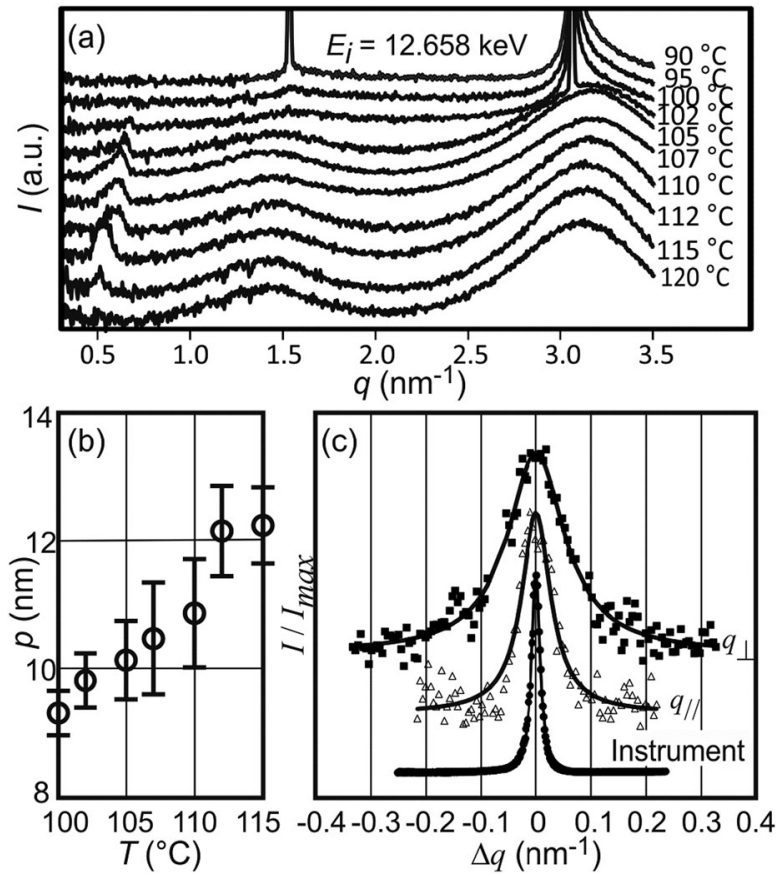

Fig. 2 (a) The resonant diffraction peak is only observed in the $N_{\text {tb }}$ phase of Se45. (b) The helical pitch $p$ of the $\mathrm{N}_{\text {tb }}$ phase decreases with cooling. (c) Fit of a Lorentzian profile to the resonant diffraction peak of the $\mathrm{N}_{\mathrm{tb}}$ phase, recorded at $112{ }^{\circ} \mathrm{C}$.

phase and at the biphasic boundaries. As shown in Fig. 2a, the resonant peak appeared at the onset of the $\mathrm{N}_{\mathrm{tb}}$ phase $\left(115^{\circ} \mathrm{C}\right)$, reaching a maximum intensity at $112{ }^{\circ} \mathrm{C}$, with $p=12.1 \mathrm{~nm}$. With decreasing temperature, the resonant peak shifted to larger $q$, indicating a reducing pitch length (Fig. 2b). The radial full width at half maximum (FWHM) of the resonant peak initially increased, indicating a broadening distribution of $p$, before decreasing again at the onset of the SmC phase. The resonance effect was lost with further cooling into the SmC phase. These findings are summarised numerically in Table S2 (ESI $\dagger$ ).

At $112{ }^{\circ} \mathrm{C}$, the sharp resonant peaks were exactly on the meridian (Fig. 3a and b), defined as the axis through the beam centre, parallel to the magnetic field. This indicates that the helical axis of the bulk sample was highly oriented with the field. The diffuse SAXS peaks were azimuthally spread (arc-like), but were also centred on the meridian (Fig. 3a-c). As mentioned above, these SAXS features arise from local smectic-like layering of dimers. The azimuthal arcing of the SAXS maxima indicates that the short-range layers are not all perpendicular to the helical axis.

On cooling from $112{ }^{\circ} \mathrm{C}$, the intensity maxima of all three SAXS peaks simultaneously shifted away from the meridian; the lower the temperature, the larger the shift, see Fig. 3b-d. The resonant peak began to broaden azimuthally, indicating decreasing orientational order of the $\mathrm{N}_{\mathrm{tb}}$ phase. Phase orientation was lost entirely in the SmC phase - see the sharp Bragg ring in Fig. 3d. The lamellar phase is assumed to be SmC (tilt angle $\sim 24^{\circ}$ ) because the layer height $(4.12 \mathrm{~nm}$ ) is shorter than the length of both dimer types in the mixture. The WAXS

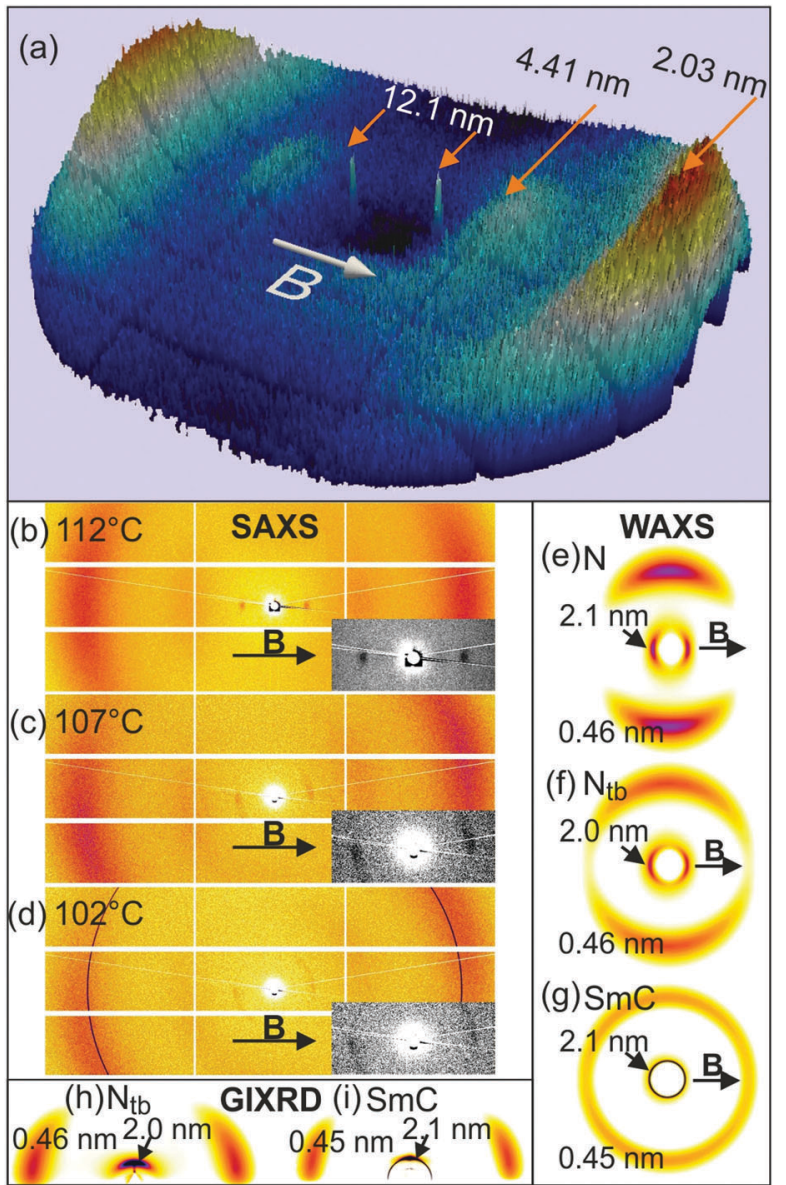

Fig. 3 (a) Surface plot of the SAXS diffractogram of the $N_{\text {tb }}$ phase of Se45 at $112{ }^{\circ} \mathrm{C}$ aligned in a magnetic field, $E_{\mathrm{i}}=12.658 \mathrm{keV}$. (b-d) 2D SAXS patterns of the $\mathrm{N}_{\text {tb }}$ phase at $112{ }^{\circ} \mathrm{C}, 107^{\circ} \mathrm{C}$ and $102{ }^{\circ} \mathrm{C}$, with zoom-in of the sharp resonant SAXS peaks. (e-g) Transmission WAXS patterns of Se45 in the (e) N, (f) $N_{\text {tb }}$ and (g) SmC phases. (h and i) Wide-angle GIXRD patterns of $\mathrm{Se} 45$ in the (h) $\mathrm{N}_{\mathrm{tb}}$ and (i) SmC phases; smectic layers are parallel to the horizontal substrate.

maxima similarly rotated and spread azimuthally, before becoming a diffuse halo in the SmC (Fig. 3e-g).

The temperature dependency of the pitch length was reported by C. Zhu et al., ${ }^{11}$ who measured $p$ of CB7CB to vary between 8 and $10 \mathrm{~nm}$. However in their work, the reflection was usually split over a wider $q$-range, possibly due to a surface confinement effect. The increased $p$ of Se $45(9-12 \mathrm{~nm})$ is attributed to the larger molecular lengths of DTC5C7 and DTSe.

\section{Structural correlation and orientational order}

At $112{ }^{\circ} \mathrm{C}$, the azimuthal FWHM of the resonant peak was $\sim 12^{\circ}$, indicating that the orientational order parameter of the global helical axis $\left(S_{0}\right)$ must be close to $1\left(\left(3 \cos ^{2} 6^{\circ}-1\right) / 2=0.98\right.$, taking $12^{\circ} / 2=6^{\circ}$ as the mean deviation angle of the local helical directors to the external field). This is in fact an underestimation as it assumes no contribution from other peak broadening 
factors, such as the structural correlation lengths. However, the mesogens are tilted (tilt angle $\theta$ ) in respect to the helical axis, which means that a second order parameter $S_{1}=\left\langle 3 \cos ^{2} \theta-1\right\rangle / 2$ is required to account for this angular distribution. The overall orientational order parameter $S$ of the $\mathrm{N}_{\mathrm{tb}}$ phase, measureable by classical means, is therefore $S_{0} \cdot S_{1}$, which quantifies the orientational distribution of the helical domains and the tilt angle distribution of the mesogens inside each domain. We have shown that $S_{0}$ of the $\mathrm{N}_{\mathrm{tb}}$ phase can be close to 1 and that it decreases with temperature, but even in the case of perfect helical alignment, the classically measured orientational order parameter will be small because $S_{1} \ll 1$, due to $\theta \gg 0^{\circ}$ (see next section on the molecular-level model and Fig. 4). The fundamental limitation imposed by $S_{1}$ may account for the low
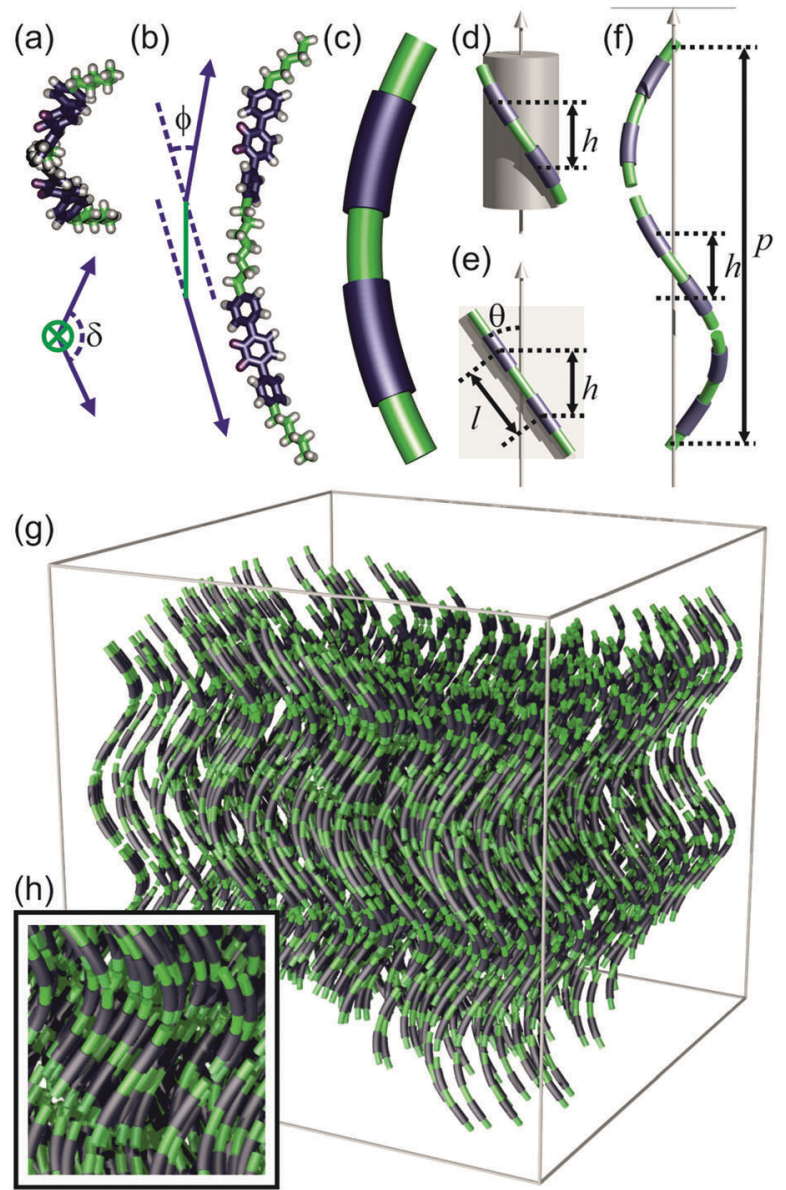

Fig. 4 Model of the $N_{t b}$ phase. ( $a$ and $b$ ) A bent dimer in a conformation resembling a segment of a helix; (a) projection along the spacer axis, and the twist angle of the dimer $\delta$ is defined as the angle between the axis of the two mesogens on projection (represented by blue arrows). (b) Side view, where the bend angle of the dimer $\phi$ is the angle between the axis of the two mesogens (blue arrows) linked by the spacer (green line). (c) Simplified representation of the ideal molecular conformation (average of different conformations, and of DTC5C7 and DTSe) as a helical segment. (d) A dimer wraps around a cylinder. $h=$ local layer height. (e) An "unwrapped" dimer. $\theta=$ tilt angle, $l=$ contour length between centres of mesogens. (f) Local arrangement of dimers along the helical axis. $p=$ pitch. (g) $N_{\text {tb }}$ phase assembled from molecules with average conformations approximating helical segments; local layering is shown in (h). orientational order parameter $(\langle P 2\rangle \sim 0.4-0.6)$ often reported within the $\mathrm{N}_{\mathrm{tb}}$ phase of bent dimers. ${ }^{8-10,14-16}$

Assuming perfect orientational order of the helical axis (reasonable at $112{ }^{\circ} \mathrm{C}$ where $S_{0} \simeq 1$ ), the intensity distribution of the resonant peak can be attributed to structural correlations parallel and perpendicular to the helical axis. In a nematic LC, one may assume that correlation decays exponentially with distance $r$, i.e. $\propto \exp (-r / \xi)$. Under this assumption, the scattered intensity in reciprocal space is well reproduced by Lorentzian profiles of the form $I_{\max } /\left(1+\Delta q_{\|}{ }^{2} \xi_{\|}{ }^{2}+\Delta q_{\perp}{ }^{2} \xi_{\perp}{ }^{2}\right),{ }^{30-32}$ where $\xi_{\|}$ and $\xi_{\perp}$ are the correlation lengths parallel and perpendicular to the helical axis. By least squares fitting of a Lorentzian profile to the intensity around the resonant peak at $112{ }^{\circ} \mathrm{C}$ ( $I$ vs. $\Delta q_{\|}$and $I$ vs. $\Delta q_{\perp}$ ), and after making corrections for instrumental broadening (Fig. 2c), we obtained $\xi_{\|}=33 \mathrm{~nm}$ and $\xi_{\perp}=18 \mathrm{~nm}$. Alternatively, using the FWHM of the Lorentzian fit $(\Delta q=2 / \xi)$, the Scherrer equation $\tau=2 K \pi / \Delta q$ can be used to estimate the size of the coherent heliconical domain. If the shape factor $(K)$ is set to unity, then $\tau=\pi \xi$ and the longitudinal and transverse helical domain sizes are estimated to be $104 \mathrm{~nm}$ (24 molecular lengths) and $57 \mathrm{~nm}$ (112 molecular widths). Note that both values are underestimates: the calculations assumed that the breadth of the resonant peak arose entirely from structural correlation. However, the distribution of $p$ and the orientational imperfection also contribute to the peak breadth, as discussed above. At temperatures below $112{ }^{\circ} \mathrm{C}, \xi_{\perp}$ can no longer be determined due to the increased contribution of $S_{0} \ll 1$ to azimuthal peak broadening. Similarly the increasing distribution of $p$ with reducing temperature prevents accurate measurement of $\xi_{\|}$. However it would appear that $\xi_{\|}$reduces as the $\mathrm{N}_{\mathrm{tb}}$ phase progresses, until reaching the onset of the SmC phase, where the quasi-long-range positional order sets in ref. 33. For the SmC phase, measurement of $\Delta q_{\| d 2}$ is limited by the instrumental resolution $\left(0.005 \mathrm{~nm}^{-1}\right)$, indicating a correlation length $>400 \mathrm{~nm}$.

As mentioned previously, the two broad SAXS peaks are respectively linked to short-range correlations between the dimers and mesogens. These correlations must be (on average) in the direction of the helical axis because the two broad SAXS peaks remain aligned with the resonant peak at all times, despite the reducing influence of the magnetic field with cooling. At temperatures close to the $\mathrm{N}-\mathrm{N}_{\mathrm{tb}}$ boundary, the inter-mesogen correlation length along the helical axis can be estimated from $2 / \Delta q_{\| d 2}$ (Table S2, ESI $\dagger$ ) as $\sim 1.7 \mathrm{~nm}$.

\section{Molecular-level model}

The existing models of the $\mathrm{N}_{\mathrm{tb}}$ phase describe an average helical director field inside a nematic continuum, but lack consideration of local molecular packing. Here, we propose a locally layered molecular-level model of the $\mathrm{N}_{\mathrm{tb}}$ phase, in line with the newly presented information discussed above. In this model, the ideal average conformation of the molecules (DTC5C7 and DTSe) resembles the shape of a helical segment (Fig. 4a-c), which enables each component of the dimer to 
match the heliconical path of the $\mathrm{N}_{\mathrm{tb}}$ director field (Fig. 4f). In the real dynamic system, there is a multitude of conformations. However in the following geometric construction we assume, for simplicity, the molecular conformation that follows the ideal shape of a helical segment, representing the conformational average, and show that it is energetically feasible.

The local director field along the helical axis can be defined mathematically as:

$$
\mathbf{n}=\sin \theta \cos (-2 \pi z / p) \boldsymbol{i}+\sin \theta \sin (-2 \pi z / p) \boldsymbol{j}+\cos \theta \boldsymbol{k}
$$

where $z$ is the height along the helical axis, $\theta$ is the tilt angle of the mesogens to the helical axis and $p$ is the pitch length of the resulting helix. On progression along $z$, the dimers can be imagined to wrap around a cylinder, such that each successive mesogen is rotated about the helical axis by $2 \pi h / p$ (Fig. $4 \mathrm{~d}-\mathrm{f}$ ), where $h$ denotes the cybotactic layer height, i.e. $h \cong d 2$ (Table $\mathrm{S} 2, \mathrm{ESI} \dagger)$. The tilt angle of the mesogens can also be calculated using the relation:

$$
h=l \cos (\theta)
$$

where $l$ is the contour length between the centres of the two mesogens of each dimer $(\sim 2.3 \mathrm{~nm})$ (average of DTC5C7 and DTSe, see ESI $\dagger$ for details). This equation can in fact be used for the nematic phase too, where the constant $\cos (\theta)$ of the $\mathrm{N}_{\mathrm{tb}}$ phase should be replaced by an average value $\langle\cos (\theta)\rangle$. In the $\mathrm{N}$ phase, $\theta$ becomes the tilt angle of molecules towards the molecular director instead of the helical axis, and it changes very little at the $\mathrm{N}-\mathrm{N}_{\mathrm{tb}}$ transition, as indicated by a similar value of $d 2$ in the two phases. As shown in Table S2 (ESI $\dagger$ ), the value of $h$ is almost invariant with temperature $(\sim 2 \mathrm{~nm})$, suggesting a near constant tilt angle of $\sim 29^{\circ}$ in the $\mathrm{N}_{\text {tb }}$ phase. This tilt angle is larger than that obtained from birefringence measurements on DTC5C7, ${ }^{12}$ but is broadly in line with those discussed for CBCnCB systems; ${ }^{14}$ it is also slightly larger than the dimer tilt angle estimated for the SmC phase. A smaller tilt in the $\mathrm{SmC}$ is in fact suggested by GIXRD experiments on oriented thin films of Se45 (Fig. 3h and i); this is based on the smaller azimuthal spread in WAXS and the larger $d$-spacing in SAXS, found in the SmC phase.

Using the experimental values of the rotation and tilt angles, one may also calculate the bend angle between the two mesogens of a dimer. If the two rod-like mesogens are respectively defined by unit vectors $\mathbf{n}_{1}$ and $\mathbf{n}_{2}$ (see Fig. S7), it can be shown geometrically $(\mathrm{ESI} \dagger)$ that:

$$
\sin (\phi / 2)=\sin (\theta) \cdot \sin (\pi h / p)
$$

where $\phi$ is the exterior bend angle between them (for a linear dimer $\phi=0$, Fig. 4b). At $115{ }^{\circ} \mathrm{C}$, $\phi$ was calculated to be $28^{\circ}$, which increased to $35^{\circ}$ on cooling to $105{ }^{\circ} \mathrm{C}$. In both instances, $\phi$ is surprisingly low and much smaller than the bend angle imposed by an all-trans spacer $\left(>60^{\circ}\right)$.

For the dimer molecules, another important parameter is its twist angle $\delta$, i.e. the angle between the two mesogens when projected along the spacer axis (Fig. 4a). Using our model, it could be calculated that the twist angle was $129^{\circ}$ at $115{ }^{\circ} \mathrm{C}$, and $125^{\circ}$ at $105{ }^{\circ} \mathrm{C}$ (Section S5.3, ESI $\dagger$ ). These values are far from the $0^{\circ}$ twist angle for an all-trans conformation of the spacer.

In comparison to our model, a molecular dynamic simulation of the $\mathrm{N}_{\mathrm{tb}}$ phase formed by the cyanobiphenyl dimer CB7CB was carried out by D. Chen et al. in a periodic bounding box of size $5.6 \times 5.6 \times 8.0 \mathrm{~nm} .^{7}$ According to this, the average external bend angle $\phi$ of the dimer was $\sim 47^{\circ}$, significantly larger than estimated from our model of Se45. Vanakaras and Photinos $^{34}$ used a very simplified molecular model of two rod-like units (representing the two mesogens in the dimer) with a fixed core-core distance between them, and predicted that a bend angle of $\sim 60^{\circ}$ and a twist angle also of $\sim 60^{\circ}$ would be best for the formation of the $\mathrm{N}_{\mathrm{tb}}$ phase.

The results presented here (low bending angle and high twist angle of the dimer) suggest that in order to follow a heliconical path, the molecules must adopt a higher energy conformation in which the arms are twisted about the spacer axis and are closer to parallel than in the minimum energy conformation of an isolated molecule. Molecular modelling (ESI, $\uparrow$ Section S6) has shown that this can be achieved by changing the torsion angles of $\mathrm{C}-\mathrm{C}$ bonds in the spacer, such as the molecule shown in Fig. $4 \mathrm{a}$ and $\mathrm{b}$. In the $\mathrm{N}_{\mathrm{tb}}$ phase, it is likely that this distortion energy is compensated by the entropy associated with the retained positional freedom of the dimers. The increasing $\phi$ on cooling is therefore expected, as lower energy conformations are preferred at lower temperatures. Furthermore, eqn (3) shows that $p \propto 1 / \phi$. On cooling, as $\phi$ increases, the helical pitch $p$ is reduced. On further cooling, when the translational entropy gain becomes insufficient to outweigh the energy cost of the distortion of the spacer, the system transforms to the SmC. The true nature of this smectic phase is still under investigation. There would be little advantage in chirality synchronization if it turns out to be a standard SmC phase, as longitudinal translational contribution to entropy is minor in a smectic anyway. A further likely consequence of the continuous change in helical pitch on cooling is a breakup into smaller domains, resulting in loss of the initial high orientational order of the helical axis, and thus also resulting in poor alignment in the SmC.

At the high- $T$ end, as $\phi$ decreases and the dimer straightens further, the helix unwinds at the $\mathrm{N}_{\mathrm{tb}}-\mathrm{N}$ transition as longitudinal motion no longer requires the molecules to follow the helical path. The drop in $\phi$ at the transition is indicated by the reduction in azimuthal spread of the WAXS arcs in Fig. 3e and $\mathrm{f}$.

The establishment of long-range homochirality in the $\mathrm{N}_{\mathrm{tb}}$ phase appears to be a unique example of entropy-driven chirality synchronisation in achiral systems. Chirality synchronisation occurs where cooperativity in the choice of same-sense conformation in many molecules lowers the free energy, see recent reviews. ${ }^{35,36}$ As far as we know, in all other reported cases the reduction in free energy means in fact lowering the interaction energy through closer packing.

In summary, using a specially synthesised selenium-containing compound, we have presented the first RXS experiment on the $\mathrm{N}_{\mathrm{tb}}$ phase using hard X-rays, which allowed sample alignment. Resonant Bragg reflection at Se absorption edge revealed a temperature dependent helical pitch of 9-12 nm. Of the two 
orientational order parameters required for the $\mathrm{N}_{\mathrm{tb}}$ phase, we found that of the helical axis, measured here for the first time, to reach an unprecedented value of $S_{0}=0.98-1.00$. Simultaneous recording of the resonant and the higher $q$ non-resonant scattering, impossible with carbon RXS, and the knowledge that the non-resonant features come from perfectly aligned helices, allowed us to determine the tilt angle of the mesogens relative to the helical axis, as well as the bend angle of the dimers. We thus obtained a molecular-level model of the $\mathrm{N}_{\mathrm{tb}}$ phase. According to this, the mesogen arms of the dimer are closer to parallel than in the minimum energy conformation, and are twisted about the spacer axis. Furthermore we found that, while the segregation of the aromatic and aliphatic moieties in layers is only local, the coherently diffracting heliconical domains size is of the order of $0.1 \mu \mathrm{m}$.

\section{Acknowledgements}

We acknowledge funding from the joint NSF-EPSRC PIRE program "RENEW" (EP/K034308), the Leverhulme Trust (RPG-2012-804) and the EPSRC (EP/M015726) and (EP/J004480) for ZA and CW and the EPSRC NMSF, Swansea for high resolution mass spectra. GU is grateful for the award of State Specially Recruited Expert from the Government of China. For help with the synchrotron experiments we thank Prof. N. Terrill at I22, Diamond Light Source, and Drs O. Bikondoa, L. Bouchenoire, S. Brown, P. Thompson and D. Wermeille of the XMaS beamline BM28 at ESRF.

\section{References}

1 G. Ungar, V. Percec and M. Zuber, Macromolecules, 1992, 25, 75.

2 G. Ungar, V. Percec and M. Zuber, Polym. Bull., 1994, 32, 325.

3 V. P. Panov, R. Balachandran, M. Nagaraj, J. K. Vij, M. G. Tamba,

A. Kohlmeier and G. H. Mehl, Appl. Phys. Lett., 2011, 99, 261903.

4 C. Meyer, G. R. Luckhurst and I. Dozov, Phys. Rev. Lett., 2013, 111, 067801.

5 E. Gorecka, M. Salamonczyk, A. Zep, D. Pociecha, C. Welch, Z. Ahmed and G. H. Mehl, Liq. Cryst., 2015, 42, 1.

6 V. Borshch, Y.-K. Kim, J. Xiang, M. Gao, A. Jákli, V. P. Panov, J. K. Vij, C. T. Imrie, M. G. Tamba, G. H. Mehl and O. D. Lavrentovich, Nat. Commun., 2013, 4, 2635.

7 D. Chen, J. H. Porada, J. B. Hooper, A. Klittnick, Y. Shen, M. R. Tuchband, E. Korblova, D. Bedrov, D. M. Walba, M. A. Glaser, J. E. Maclennan and N. A. Clark, Proc. Natl. Acad. Sci. U. S. A., 2013, 110, 15931.

8 L. Beguin, J. W. Emsley, M. Lelli, A. Lesage, G. R. Luckhurst, B. A. Timimi and H. Zimmermann, J. Phys. Chem. B, 2012, 116, 7940.

9 Z. Zhang, V. P. Panov, M. Nagaraj, R. J. Mandle, J. W. Goodby, G. R. Luckhurst, J. C. Jones and H. F. Gleeson, J. Mater. Chem. C, 2015, 3, 10007.

10 K. Adlem, M. Čopič, G. R. Luckhurst, A. Mertelj, O. Parri, R. M. Richardson, B. D. Snow, B. A. Timimi, R. P. Tuffin and D. Wilkes, Phys. Rev. E: Stat., Nonlinear, Soft Matter Phys., 2013, 88, 022503.
11 C. Zhu, M. R. Tuchband, A. Young, M. Shuai, A. Scarbrough, D. M. Walba, J. E. Maclennan, C. Wang, A. Hexemer and N. A. Clark, Phys. Rev. Lett., 2016, 116, 147803.

12 N. Sebastián, M. G. Tamba, R. Stannarius, M. R. de la Fuente, M. Salamonczyk, G. Cukrov, J. Gleeson, S. Sprunt, A. Jákli, C. Welch, Z. Ahmed, G. H. Mehl and A. Eremin, Phys. Chem. Chem. Phys., 2016, 18, 19299.

13 S. A. Pardaev, S. Shamid, M. G. Tamba, C. Welch, G. H. Mehl, J. T. Gleeson, D. Allender, J. V. Selinger, B. D. Ellman, A. Jakli and S. Sprunt, Soft Matter, 2016, 12, 4472.

14 C. Meyer, G. R. Luckhurst and I. Dozov, J. Mater. Chem. C, 2015, 3, 318.

15 V. P. Panov, M. Nagaraj, J. K. Vij, Yu. P. Panarin, A. Kohlmeier, M. G. Tamba, R. A. Lewis and G. H. Mehl, Phys. Rev. Lett., 2010, 105, 167801.

16 A. Hoffmann, A. G. Vanakaras, A. Kohlmeier, G. H. Mehl and D. J. Photinos, Soft Matter, 2015, 11, 850.

17 S. M. Salili, C. Kim, S. Sprunt, J. T. Gleeson, O. Parri and A. Jákli, RSC Adv., 2014, 4, 57423.

18 M. G. Tamba, S. M. Salili, C. Zhang, A. Jákli, G. H. Mehl, R. Stannarius and A. Eremin, RSC Adv., 2015, 5, 11207.

19 P. A. Henderson and C. T. Imrie, Liq. Cryst., 2011, 38, 1407. 20 M. Cestari, E. Frezza, A. Ferrarini and G. R. Luckhurst, J. Mater. Chem., 2011, 21, 12303.

21 P. K. Challa, V. Borshch, O. Parri, C. T. Imrie, S. N. Sprunt, J. T. Gleeson, O. D. Lavrentovich and A. Jákli, Phys. Rev. E: Stat., Nonlinear, Soft Matter Phys., 2014, 89, 060501.

22 M. Cestari, S. Diez-Berart, D. A. Dunmur, A. Ferrarini, M. R. de la Fuente, D. J. B. Jackson, D. O. Lopez, G. R. Luckhurst, M. A. Perez-Jubindo, R. M. Richardson, J. Salud, B. A. Timimi and H. Zimmermann, Phys. Rev. E: Stat., Nonlinear, Soft Matter Phys., 2011, 84, 031704.

23 R. Meyer, in Molecular Fluids, ed. R. Balian and G. Weil, Gordon and Breach, New York, 1976.

24 I. Dozov, Europhys. Lett., 2001, 56, 247.

25 R. Memmer, Liq. Cryst., 2002, 29, 483.

26 D. H. Templeton and L. K. Templeton, Acta Crystallogr., 1980, A36, 237-241.

27 W. A. Hendrickson, Science, 1991, 254, 51.

28 J.-L. Hodeau, V. Favre-Nicolin, S. Bos, H. Renevier, E. Lorenzo and J.-F. Berar, Chem. Rev., 2001, 101, 1843.

29 A. de Vries, Mol. Cryst. Liq. Cryst., 1970, 10, 219.

30 G. J. Brownsey and A. J. Leadbetter, Phys. Rev. Lett., 1980, 44, 24.

31 K. K. Chan, P. S. Pershan and L. B. Sorensen, Phys. Rev. A: At., Mol., Opt. Phys., 1986, 34, 2.

32 S. H. Hong, R. Verduzco, J. C. Williams, R. J. Twieg, E. DiMasi, R. Pindak, A. Jákli, J. T. Gleeson and S. Sprunt, Soft Matter, 2010, 6, 4819.

33 P. S. Pershan, Structure of Liquid Crystal Phases, World Scientific Publishing Ltd, 1988.

34 A. G. Vanakaras and D. J. Photinos, Soft Matter, 2016, 12, 2208.

35 C. Tschierske and G. Ungar, ChemPhysChem, 2016, 17, 9.

36 E. Yashima, N. Ousaka, D. Taura, K. Shimomura, T. Ikai and K. Maeda, Chem. Rev., 2016, 116, 13752. 